\title{
TTR
}

Traduction, terminologie, re?daction

\section{Littérature et diglossie : créer une langue métisse ou la " chamoisification " du français dans Texaco de Patrick Chamoiseau}

\section{Marie-José N’Zengou-Tayo}

Volume 9, numéro 1, 1er semestre 1996

Le festin de Babel

Babel's Feast

URI : https://id.erudit.org/iderudit/037243ar

DOI : https://doi.org/10.7202/037243ar

Aller au sommaire du numéro

Éditeur(s)

Association canadienne de traductologie

ISSN

0835-8443 (imprimé)

1708-2188 (numérique)

Découvrir la revue

Citer cet article

N’Zengou-Tayo, M.-J. (1996). Littérature et diglossie : créer une langue métisse ou la " chamoisification » du français dans Texaco de Patrick Chamoiseau. TTR, 9(1), 155-176. https://doi.org/10.7202/037243ar
Résumé de l'article

Littérature et Diglossie : créer une langue métisse ou la " chamoisification » du français dans Texaco de Patrick Chamoiseau - Le succès de Texaco et les commentaires élogieux concernant l'usage du français et du créole par l'auteur invitent à examiner de plus près le jeu linguistique à l'oeuvre dans le roman en prenant en compte les positions théoriques avancées par Bernabé, Chamoiseau et Confiant dans Éloge de la créolité (1989). Bien que le roman s'inscrive dans une tradition de l'utilisation littéraire du créole fort ancienne, il est possible d'identifier la spécificité de l'écriture de Chamoiseau. Pratiquant une poétique de "l'inquiétante étrangeté », il crée un " effet-de-créole " grâce auquel il déroute et séduit ses lecteurs hexagonaux aussi bien qu'antillais. Placée sous le signe du Baroque, cette écriture est devenue le symbole du métissage culturel et/ou du processus de créolisation, caractéristique des cultures de la région des Caraïbes et riche de la promesse d'un nouveau rapport-au-monde basé sur le plurilinguisme.
Tous droits réservés (C) TTR: traduction, terminologie, rédaction — Les auteurs, 1996
Ce document est protégé par la loi sur le droit d'auteur. L'utilisation des services d'Érudit (y compris la reproduction) est assujettie à sa politique d'utilisation que vous pouvez consulter en ligne. 


\section{Littérature et diglossie : créer une langue métisse ou la " chamoisification " du français dans Texaco de Patrick Chamoiseau}

\section{Marie-José N'Zengou-Tayo}

À sa parution en 1992, Texaco fut salué avec enthousiasme par la critique parisienne comme l'événement de la rentrée littéraire et se vit décerner le prix Goncourt. L'écrivain tchęque Milan Kundera était parmi les premiers à célébrer ce roman. Ce qu'il exaltait le plus dans l'œuvre, c'était l'usage de la langue française qu'y faisait Chamoiseau. Dans un article antérieur, le romancier avait décrété, en effet, qu'il ne s'agissait ni d'un français créolisé, ni d'un créole francisé, mais d'un français " chamoisisé " (Kundera, 1991). Un lecteur familier d'Éloge de la créolité (1989) reconnaîtra ici l'allusion à une des déclarations du trio Bernabé, Chamoiseau, Confiant qui exprimaient leur refus de jouer d'une créolisation du français ou d'une francisation du créole :

Ce ne sera pas forcément du français créolisé ou réinventé, du créole francisé ou réinventé, mais notre parole retrouvée et finalement décidée. Notre singularité exposée - explosée dans l'Être. (p. 46)

Cependant, sachant que le va-et-vient d'une langue à l'autre produit nécessairement une contamination linguistique, on ne peut que s'interroger sur la faisabilité d'un tel projet. Par ailleurs, le créole martiniquais n'étant pas fixé (pas d'orthographe officielle ni de dictionnaire monolingue), comment peut-on être sûr qu'il ne s'agit 
pas d'un créole " réinventé » ? Dans cet article, nous chercherons à identifier les différents modes d'utilisation du créole dans le roman Texaco. Nous essayerons d'évaluer jusqu'à quel point Chamoiseau parvient à concrétiser, dans son roman, le projet linguistique annoncé dans Éloge de la créolité, ainsi que la viabilité d'un tel projet.

Avant d'entamer notre analyse, il nous semble nécessaire de rappeler le statut du créole dans la plupart des sociétés antillaises et dans la société martiniquaise en particulier. Le sociologue haîtien Jean Casimir remarque dans son essai, la Caraibe une et divisible (1991), que

[1] communication parlée reproduit la nature particulière des relations entre colonisés et colonisateurs. Les échanges sociaux qui échappent à l'imposition coloniale - vie privée en tout premier lieu - sont réservés aux langues créoles. D'autres activités sociétales sont l'apanage de la langue du maitre. (p. 121)

De même, le linguiste Chaudenson (1989) note que

[1]es DOM [Départements d'Outre-Mer] présentent des situations qu'on peut qualifier [...] de " diglossiques " en marquant par là que se retrouvent, dans la même aire et au sein de la même communauté linguistique, deux langues (le français et un créole) de statut social inégal et de répartition fonctionnelle en gros complémentaire (le français dans les situations formelles et/ou publiques; le créole dans les situations informelles et/ou privées). (p. 192)

En Martinique, la question des deux langues est ressentie avec encore plus d'acuité par les intellectuels et les écrivains, qui en font souvent mention dans leurs écrits, notamment les écrivains de la Créolité. Ces derniers vivent de façon encore plus intense que leurs aînés de la Négritude la dichotomie créole-français et leur rapport à ces deux langues car, comme le montre l'analyse de certains linguistes antillais; la notion de diglossie est fortement rattachée à la 
situation coloniale (Prudent, 1981) et aux préjugés linguistiques qu'elle a contribués à véhiculer. Il faut donc lire les déclarations des auteurs d'Éloge et replacer leur pratique d'écriture dans ce contexte idéologique :

La littérature créole d'expression française aura donc pour tâche urgente d'investir et de réhabiliter l'esthétique de notre langage. C'est ainsi qu'elle sortira de l'usage contraint du français qui, en écriture, a trop souvent été le nôtre. (p. 46)

Effectivement, ces écrivains déploient diverses stratégies pour tenter de résoudre la question linguistique antillaise au niveau de l'écriture. En affichant la recherche du contact interlinguistique pour " amplifier l'audience d'une connaissancelittéraire [d'eux]-mêmes " (p. 48), ils lancent un défi à la création artistique. Ce faisant, leur écriture s'inscrit dans le champ du baroque et il nous semble qu'elle traduise une exploration des possibilités qu'offre ce style dans le cadre du plurilinguisme antillais.

Le jeu entre plusieurs langues (leurs lieux de frottements et d'interactions) est un vertige polysémique. Là se trouve le canevas d'un tissu allusif, d'une force suggestive, d'un commerce entre deux intelligences. Vivre en même temps la poétique de toutes les langues, c'est non seulement enrichir chacune d'elles, mais c'est surtout rompre l'ordre coutumier de ces langues, renverser leurs significations établies. C'est cette rupture qui permettra d'amplifier l'audience d'une connaissance littéraire de nous-mêmes. (p. 48)

Ce projet esthétique, nous le voyons à l'œuvre dans Texaco. Il reste cependant à identifier les mécanismes par lesquels Chamoiseau emporte son lecteur dans " un vertige polysémique ", repérer " le frottement " entre les deux langues ainsi que la rupture de leur " ordre coutumier" et le renversement « des significations établies ». Notons au passage les dimensions nietzschéenne et mallarméenne de ce projet qui vise à « renverser le code des signes 
quotidiens 》 (Nietzsche)' et "à donner un sens plus pur aux mots de la tribu n, selon le mot de Mallarmé.

Signalons, toutefois, que l'utilisation littéraire du créole et du français n'est pas chose nouvelle. Qu'il le veuille ou non, Chamoiseau est l'héritier d'une tradition littéraire qui a tenté elle aussi de jouer sur la diglossie. Le premier texte poétique créole, " Lisette quitté la plaine ", a été transcrit par Moreau de Saint-Méry, Créole guadeloupéen, auteur de la Description de la partie française de Saint-Domingue (1797-1798), dès le XVIII 'siècle. L'insertion du créole dans des romans en français se pratique au tout début $\mathrm{du}_{\mathrm{XX}} \mathrm{C}^{\circ}$ siècle dans la littérature haïtienne, par exemple. Ainsi, dans la Famille des Pitite-Caille et dans Zoune chez sa nainnaine de Justin Lhérisson (1906), lés citations en créole sont italicisées mais présentées sans traduction. Ces occurrences créoles sont liées à l'esthétique réaliste du roman de cette époque. Le créole est utilisé avec une double fonction de recherche d'authenticité (parler des gens du peuple, Zoune) d'une part, et de satire sociale d'autre part (les nouveaux-riches trahissent leurs origines populaires par l'usage du créole, la Famille). On ne peut parler d'exotisme puisque ces textes ne visent pas le public étranger (absence de traduction) et sont publiés en feuilleton dans la presse locale ${ }^{2}$. L'utilisation littéraire du créole a souvent suivi ce modèle jusqu'à la parution, en 1945, de Gouverneurs de la rosée de Jacques Roumain. Dans ce roman, Roumain pratique une esthétique considérée comme " révolutionnaire » en pratiquant un glissement continu du français courant au français " créolisé ". Cette technique a été reprise de façon encore plus systématique par la Guadeloupéenne Simone Schwarz-Bart dans Pluie et vent sur Télumée-Miracle (1971) et Ti Jean L'horizon $(1979)^{3}$.

1. Cité par Pierre Klossowski (1969).

2. Cf. Prudent (1981), p. 34, sur la Famille des Pitite-Caille (1906) du même auteur.

3. Cf. l'analyse de Jean Bernabé (1978 et 1979). 
Que faut-il entendre par " français créolisé " ? Du point de vue linguistique, il me semble qu'on peut parler de crélisation du français, lorsque un texte présenté comme « français » (orthographe, lexique, syntaxe) contient un certain nombre de tournures (syntaxe, lexique) qui ne sont pas originelles et appartiennent manifestement à une langue différente (le créole). Pour les langues créoles contenant un substrat français, la question se pose de savoir comment repérer un mot créole dont l'orthographe aurait été francisée. On se rend alors compte que la distance créole-français peut être aisément brouillée et qu'il est peut-être plus facile d'identifier un anglicisme qu'un créolisme. La proximité des deux langues et, en même temps, leur différence offre donc aux écrivains de la créolité la possibilité de pratiquer une stylistique de " l'étrangeté créole " (Chamoiseau, 1994, p. 156) qui dérange dans la mesure où le lecteur français (non-créolophone) se retrouve face à un texte dont la facture (la texture) est apparemment française (le familier) mais dont le sens et la structure lui sont étrangers (l'étrange). C'est sans doute ainsi qu'il faut interpréter la notion d'opacité que revendiquent ces écrivains, dans le sillage de Glissant (1989).

Notre plongée dans la Créolité ne sera pas incommunicable mais elle ne sera non plus pas totalement communicable. Elle le sera avec ses opacités, l'opacité que nous restituons aux processus de la communication entre les hommes. (p. 53)

Pourtant, s'il est plus difficile d'établir une ligne de partage nette entre les deux langues, il n'en reste pas moins possible d'identifier certaines tournures (morpho-syntaxe). La présence du créole dans un texte littéraire signale alors un écart stylistique et traduit donc la créativité de l'auteur, manipulant les deux langues pour inventer un " interlecte/idiolecte ${ }^{4}$ » ne renvoyant à aucune forme attestée dans

4. Cf. F.-L. Prudent (1981). Nous proposons cette unité «Interlecte/idiolecte» parce qu'il s'inscrit dans le cadre d'une pratique collective tout en faisant un usage personnel et original des deux langues. 
l'une ou l'autre langue. C'est en ce sens qu'il faut peut-être comprendre l'expression de Kundera parlant du français " chamoisisé ".

À l'autre extrême nous situerons l'écrivain haïtien, Frank Étienne (Frankétièn), qui écrit soit en français, soit en créole, préférant offrir une double version (traduction-réécriture)d'un même texte, comme cela s'est produit avec Dézafi (1979) / les Affres d'un déf (1980) et Pèlin tèt (1982) dont la version française a paru dans la revue Conjonction. On peut interpréter cette double production comme un refus de mélanger les deux langues s. De même, le Martiniquais Gilbert Gratiant, bien avant le mouvement de la Créolité, a toujours produit en créole ( $F a b$ Compè Zicak, 1956 et 1976 en édition bilingue).

Pour apprécier le jeu du créole et du français dans Texaco, il faut avoir présent à l'esprit les modes d'insertion du créole dans la production littéraire antillaise. Ainsi, dans une communication présentée à un colloque sur la Créolité, Pascale DeSouza (1995) distingue

[...] trois étapes caractérisant chacune une phase de l'écriture : un temps où le créole apparaît en citations uniquement, une phase d'intégration partielle du créole au français, enfin une créolisation du français. (p. 174)

et elle fait remarquer que celles-ci ne sont pas chronologiques mais correspondent à des pratiques contemporaines l'une de l'autre. Cependant, au cours de son analyse, elle se réfêre à Texaco, comme un exemple de cette troisième étape de " créolisation du français ».

5. Dès lors on ne peut qu'être sensible à l'ironie qui veut que les tenants de la Créolité signalent Dézafi comme le texte créole qui leur a révélé la puissance poétique du créole. Ils font ainsi passer au second plan l'influence de leur propre compatriote, le précurseur Gilbert Gratiant, qu'ils récuperent cependant par la suite. 
Or, la lecture de Texaco, selon sa propre grille, permet de repérer une utilisation simultanée des trois étapes (citation, intégration partielle et créolisation). Ainsi, quand l'auteur rapporte les paroles de personnages créolophones, la phrase créole est mise en italiques et suivie de sa traduction française. L'innovation de Chamoiseau vient de ce qu'il juxtapose la traduction au créole au lieu de la mettre en note de bas de page.

Alors elle m'abaissa la tête et me dit : Prédié ba papa'w ich mwen, Prie pour ton papa, mon fils... (Texaco, p. 48)

ou encore

Mé ola Matinityèz-là pasé ô-ô, Mais où est passée la Martiniquaise, oh ? (p. 248)

Il obtient ainsi un effet d'écho qui contribue, comme le fait justement remarquer DeSouza, à " renforcer [son] message " (1995, p. 182$)^{6}$. Signalons cependant certaines occurrences où le créole n'est pas traduit. Il s'agit d'un proverbe non italicisé (p. 108 : « sa ki pa bon pou zwa, pa pe bon pou kanna $n$ ), d'une expression d'un conte [scène de partage] (p. $126:$ mi ta'w, mi ta mwen, mi ta'w mi ta mwen), ou encore d'une insulte (p. 127 : Alé koké manman zòt). Ajoutons que dans certains cas la traduction donnée est assez libre. Ainsi, la phrase créole : "Man Ibo Man ho Ibo sa ta la te ye" (littéralement : Hé Mère Ibo, Mère lbo : qu'est-ce que c'était ?) devient « Madame Ibo, qu'est-ce que c'était dites donc eh bien bon dieu... " (p. 111) Ce refus d'une traduction littérale marque selon nous une volonté de souligner l'écart entre les deux langues.

6. Signalons cependant que l'italique n'est pas réservée au seul créole mais à toute " parole "/ « mot d'ordre " des personnages (autre présentation typographique du discours direct dans le roman). "Envolée la grande vie de chez les Gros-Joseph! ... Les Mathurin étaient moins fortunés " (p. 249); ou encore " [...] je lui murmurerai (pas très sure de moi mais sincère du profond) : Rabelais, mon cher... d'abord » (p. 249). 
Remarquons toutefois que Texaco rappelle l'existence de deux langues originelles absentes, d'une part le substrat africain et d'autre part le créole " pur », celui des marrons. Le substrat africain apparaît en creux dans le texte, par l'intermédiaire de la mère de Ninon, " l'impyok africaine » qui parle un " langage bâti avec les langues qu'elle avait côtoyées » (p. 109) et par celui des Mentors. Quand un groupe de quatre marrons, des Mentors, approche les anciens esclaves, ils choisissent de s'adresser à une vieille négresse Ibo. Le narrateur note cette langue « sans veut-dire » (autrement dit, sans référent sémantique), mystérieuse pour eux tous :

Le Mentor lui [la négresse Ibo] parla dans une langue sans veut dire, ou inaudible, ou bien mal prononcée, en tout cas différente. A croire qu'ils avaient tous deux vendu leur dictionnaire chrétien, elle lui répondit de la même manière, et d'une voix qui depuis charge d'années n'était plus de ce monde. (p. 111)

Notons que le dialecte africain est incompréhensible pour les nègres créolisés bien que des nègres africains vivent encore parmi eux. Ninon, par exemple, bien que très proche de sa mère, n'a pas cherché à apprendre la langue maternelle, c'est par contre cette dernière qui " mélange " différentes langues pour se faire comprendre des esclaves créoles. Dans le cas de la rencontre avec les Mentors, nous pouvons ressentir à travers les commentaires d'Esternome (" langue sans veut dire ", " vendu leur dictionnaire chrétien $»)$ toute la négativité entourant le substrat africain du créole ainsi que la dimension païenne associée à l'Afrique. Par ailleurs, le créole des Mentors se différencie de celui des békés par le rythme de la phrase et sa puissance de suggestion (p. 65).

Pour ce qui est du mélange des deux langues, il est possible de repérer le substrat créole dans Texaco à trois niveaux : celui du lexique, de la syntaxe et enfin de l'oralité. Nous détaillerons pas ici les diverses expressions et constructions utilisées par Chamoiseau tout au long des quatre-cent-vingt-six pages de son roman, la liste serait fastidieuse et la place nous manquerait. Nous avons simplement essayé d'identifier les divers procédés utilisés par 
l'auteur pour créer ce que nous avons appelé « l'effet-de-créole» pour rendre compte d'une pratique stylistique qui ne correspondra peut-être pas toujours à la production créole " réelle" des Martiniquais ordinaires.

Au niveau du lexique, la créolisation s'obtient par différents moyens. Tout d'abord, l'utilisation de mots venus de l'ancien français et passés au créole donne au lecteur francophone, mais noncréolophone, ce sentiment de l'étrange et du familier. Par exemple « bailler » (donner) qui se dit « bay » en créole, réapparait avec son orthographe étymologique tout comme "Pièce " venu de l'ancien français (aucun). De même qu'il restitue l'orthographe étymologique de certains mots créoles, Chamoiseau en transcrit d'autres avec une orthographe créolisée. Ainsi, "fouet " est orthographié "fouette " (p. 61) suivant la prononciation créole, tout comme " mulâtre " délibérément transcrit « milâtes » (p. 96) et " la croix " écrit en un seul mot " la-croix " (p. 59) pour rendre compte du phénomène d'agglutination qui s'est produit en créole.

Par ailleurs, il utilise des mots facilement reconnaissables par les francophones mais avec leur sens créole, déroutant ainsi son lecteur non-créolophone. Ainsi, "virer " du vocabulaire de la marine et passé au créole avec le sens de " tourner dans l'autre sens 》 est systématiquement utilisé là où le lecteur français s'attend à rencontrer " revenir " ou " retourner \#... "Crier " est systématiquement utilisé dans le roman avec le sens créole d'" appeler ». De même, " bagage " (" objets qu'on emporte avec soi en voyage " selon le Larousse) par exemple, signifie " chose" en créole, or, apparaissant dans une phrase comme celle-ci

Bagage bizarre, l'habitation était pour lui devenue une sorte de havre. (p. 61)

sans rien pour en élucider le sens, il donne au lecteur français le sentiment d'un rapprochement incongru entre " bagage » et " habitation ». Cette volonté de brouiller les pistes est encore plus manifeste dans un autre exemple : 
Ces deux mots portaient le même bagage (p. 85) [avaient le même sens].

où l'emploi de « porter » vise à renforcer le sens français de bagage. On comprend dès lors pourquoi Milan Kundera peut faire un clin d'ceil à Lautréamont dans l'article commentant les romans de Chamoiseau (" Beau comme une rencontre multiple "/ " Beau comme la rencontre d'un parapluie et d'une machine à coudre... ») ${ }^{7}$.

Parfois, des mots créoles sont insérés dans le texte sans référents français repérables. Nous avons relevé " sandopi " utilisé pour caractériser un homme malingre et souffreteux (Texaco, p. 22). En fait, ce décodage est possible à cause de la comparaison qui suit l'emploi de ce terme (" sandopi comme ces nègres nés sous une lune descendante... p. 22). Ailleurs, nous rencontrons le verbe " zinzoler » (pp. 62, 129) ou encore " longviller » (pp. 72, 129). Tous ces mots, indéchiffrables à première lecture, contribuent sans aucun doute à insérer dans la trame du récit ces îlots d'opacité revendiqués dans Éloge de la créolité.

Enfin, comme l'ont toujours noté les articles sur l'usage du créole en littérature, le lexique créole pénètre le texte français pour se référer aux domaines de l'espace et de la culture antillaise (faune, flore, culture traditionnelle).

Á ces utilisations citationnelles du créole viennent s'ajouter des constructions lexicales plus complexes, obtenues par suffixation (instruction > « instructionné ", p. $24=$ " qui a de l'instruction »; haillon > " haillonné ", p. $117=$ " en haillons »), agglutination (les nègres esclaves $>$ les nègresclaves, pp. $97,98,101$ ) et troncation (l'habitation $>$ " bitation $"$, pp. 61, 63, 64, 117..), dont il est difficile, dans certains cas, de décider si elles sont authentiquement créoles ou, au contraire, forgées par l'auteur, soit par analogie à

7. Cité par Delphine Perret (1995).

8. Cf. Pascale DeSouza (1995). 
d'autres formes créoles, soit en exploitant le potentiel de dérivation de la langue française. Or, il nous semble que c'est à ce niveau que fonctionne au maximum la capacité de rendement poétique du créole. Chamoiseau en joue et obtient ainsi ce que j'appelle " l'effet-de-créole ». En fait, Chamoiseau fait fonctionner le français selon un modèle générativiste " anarchique " (pour employer l'expression de Prudent (1984) concernant le fonctionnement de l'interlecte antillais), produisant des énoncés non attestés par la pratique des lecteurs mais reconnaissables par le principe d'analogie. Il crée ainsi une langue à la fois étrange et familière aussi bien pour le créolophone que le non-créolophone. Par ailleurs, étant habitué (en raison même du rapport des Antillais à la langue française) à considérer comme un créolisme tout énoncé s'écartant de la norme du français hexagonal (le " français-français» de Damas"), le lecteur créolophone est d'autant plus porté à avaliser la pratique de Chamoiseau et à " reconnaître " ces énoncés comme issus du créole $^{10}$. Comme le fait remarquer Prudent (1984) dans son article sur l'interlecte :

L'insécurité grammaticale des créolophones (et plus particulièrementdes migrants) est attestée. La force symbolique du français, langue standard de la réussite économique ne se discute pas.

9. "Hoquet ", Pigments, (1937) suivi de Névralgies, Paris, Présence Africaine, 1978.

10. Cf. Delphine Perret (1995) : " Ainsi un Martiniquais, ni littéraire ni linguiste, disait: 'Oui, on peut chercher à reconstruire le créole derrière, mais ce n'est pas facile', et un Hătien, ni littéraire ni linguiste non plus, parlait dans le même sens : 'On entend toute la musique du parler créole derrière', mais il lui était difficile d'être plus précis. " (p. 162) 
De même, le lecteur francophone, (en raison du rapport des Français à leur propre langue) est prêt à se laisser séduire par cette utilisation dérangeante, " étrange/étrangère " de sa langue ${ }^{\mathrm{il}}$.

Au niveau syntaxique, le créole imprègne le texte de Chamoiseau par l'utilisation de diverses constructions comme l'absence d'article ( " danser calenda » p. 60), la juxtaposition du complément de nom (" trous-nez » p. 82, au lieu " trous du nez » au lieu de « narine »), la suppression d'un des termes de la négation ( ( mais c'est pas le travail », p. 129). Ce dernier procédé n'est, certes, pas particulier au créole puisqu'il se retrouve dans l'emploi du registre familier du français. Il contribue cependant ici à l'effetde-créole dans la mesure où il apparaît dans une structure créolisée : "Il est vrai fout que Liberté est tout ce que tu veux, citoyen, mais c'est pas le travail » (p. 129).

On retrouve également dans le texte l'utilisation des verbes composés fragmentant l'action par un mécanisme que le linguiste Claude Hagège (1985) définit comme « une vision hyperanalytique et documentaire " (p. 38) de cette dernière. Notons sur ce point que Chamoiseau se limite à la juxtaposition de deux verbes (prendrecourir, p. 28; ramener-venir, p. 106; aller-dévirer, p. 117; appuyermonter, p. 135; etc.) alors que le créole permet des séquences plus

11. Cf. Delphine Perret (1995), p. 160. Elle rapporte la réaction de Dominique Fernandezà Texaco: « Moi-même je ne comprends pas tout ce qu'il dit, mais c'est un livre qui parle a l'imagination... " Cf. aussi Raphaell Confiant : " A mon niveau personnel, il m'aide à donner au lecteur antillais l'illusion de lire du créole. Aucun compliment ne me touche davantage que lorsqu'un lecteur me déclare avoir eu la curieuse impression d'avoir lu du créole à travers mes livres en français. Je fais donc doublement plaisir: aux Français de l'hexagone parce qu'ils retrouvent une strate profonde et oubliée de leur propre langue; aux créoles parce qu'ils ont le sentiment ou l'illusion de lire leur propre langue vernaculaire. » (Ludwig, 1994, pp. 179-180) 
longues (I feek/sot/rive/keyi yon kòk, [litt. : il vient de sortir, arriver, cueillir une noix de coco], soit : il vient tout juste de cueillir... $)^{12}$. Il modifie également le régime des verbes français. Ainsi " agriffer » est réfléchi en français, Chamoiseau l'utilise cependant dans une construction transitive directe, donnant l'impression d'une tournure créole (" le dément agriffa le béké à la gorge » (p. 56) au lieu de "le dément s'agriffa à la gorge du béké "). Il procède également à des changements de catégorie syntaxique : les verbes sont utilisés comme substantifs, les noms deviennent des adjectifs ou inversement, ou encore il combine ce changement à un glissement de sens métaphorique. Ainsi, " échassier " (n.) > " échassière " (adj.) = posée sur des échasses (p. 28). Certes, on pourra nous objecter que ces changements de catégories ne sont pas spécifiques au créole, pourtant ils se différencient de la pratique hexagonale en raison de leur contexte d'utilisation. Ainsi, lorsque nous rencontrons

(1) Aucun calculer ne leur fut ce jour-là nécessaire. (p. 22)

(2) D'être renvoyé aux champs [...] devint pour lui une crainte permanente, le raide des chatiments. (p. 55) ${ }^{13}$

nous reconnaissons la présence sous-jacente du créole dans la mesure où le français métropolitain, même argotique, n'utilisera pas ce type de construction.

Le créole se manifeste en outre par l'introduction des marques d'oralité dans le texte. Ces marques se reconnaissent par les onomatopées diverses qui émaillent la narration et par le « oui » placé en fin de phrase, et le « comme ça " si caractéristiques de la

12. Hagège (1985), p. 38. Trad. de Hagège : il m'a cueilli une noix de coco.

13. Souligné par moi. Dans le cas de " raide ", on se rend compte que l'adjectif fonctionne alors comme un synonyme du superlatif « le plus dur ». 
prosodie créole. De même, le redoublement utilisé en créole pour marquer le superlatif signale l'influence de l'oral dans le texte

[...] soudain la ligne devint molle-molle. (p. 22); un bel-beau mâle à jabot [...] (p. 23);

[...] il semblait content-content [...] (p. 64);

Liberté et Ninon se mêlèrent si tellement dans sa tête-mabolo (p. 97).

S'y ajoutent les interpellations du narrataire-auditeur-auteur(Oiseau de Cham, Chamoiseau, Ti-Cham) qui rappellent la technique du conteur martiniquais apostrophant son public au cours de sa " parole », tout en signalant la place de l'auteur dans le récit.

Comparativement à Chronique et à Solibo, Texaco représente la forme la plus aboutie du travail du style entrepris par Chamoiseau, travail beaucoup plus marqué dans ce roman que dans les deux premiers. Ainsi, Delphine Perret fait remarquer :

[...] Or si le narrateur semble alors se moquer gentiment de celui-ci [Ti-Cirique, l'Haïtien, représentant des "BellesLettres "], sa propre écriture et la " parole " rapportée de Marie-Sophie ont en fait, précisément, un caractère assez recherché. Eux aussi semblent parler comme on écrit. Qu'en penser? (Perret, 1995, p. 168)

En l'absence d'une grammaire et d'un lexique identifiant les particularismes du français de Martinique, il est difficile de faire le partage, comme nous l'avons déjà dit, entre la pratique individuelle (idiolecte) et la pratique collective (interlecte). Pourtant, si nous en croyons Prudent (1984), la pratique de l'interlecte ne se réduit pas a une somme de pratiques individuelles. Elle repose sur une " dynamique conversationnelle " (p. 44) et « se forg[e] sur un viol permanent des contraintes grammaticales des deux codes, [faisant apparaître] une jouissance d'énonciation " (p. 45). Par suite, nous pouvons considérer Texaco comme une forme expérimentale visant à inscrire dans l'espace du texte, les marques de l'oralité typiques de la narration antillaise, meilleur moyen pour l'écrivain antillais 
d'affirmer une identité littéraire sans cesse menacée par les «modèles [littéraires] obligés» (Laroche, 1991, p. 62) métropolitains.

De ce fait, il nous semble que Chamoiseau instaure un jeu entre le français et le créole, un (mé)tissage des deux langues entre les interstices desquels surgit sa propre écriture. Ce travail de l'écriture se ressent surtout au niveau des images, des comparaisons, des métaphores et d'autres figures de style articulées sur des transformations linguistiques nées de la rencontre des deux langues. Par exemple, une dévote est désignée de façon imagée par la mise en apposition du mot « ravet » utilisé aux Antilles pour désigner la blatte : " dévote ravet d'église » (p. 30). Une telle écriture se situe très clairement dans le champ du Baroque américain tel que le définissent Carlos Fuentes (1990) et Édouard Glissant (1990) et souscrit à la définition du discours romanesque " plurilingual [et] plurivocal ", selon Bakhtine (1975).

Nous pouvons donc suivre dans Texaco l'aventure de l'écrivain antillais, tentant, d'une part, de renverser le problème de la diglossie français/créole par l'exploration d'une zone interlectale et, d'autre part, d'assumer celui du rapport de l'écrit à l'oral. Pour ce faire, il invente un idiolecte à la fois étrange et familier, essayant de " mobiliser la totalité qui [lui] est offerte, tant du point de vue de l'oralité que de l'écriture " (Chamoiseau, 1994, p. 158). Il opère ainsi cette « chamoisification » du français qui a tant séduit Kundera ainsi que la presse littéraire parisienne ${ }^{14}$. La campagne médiatique ayant entouré l'attribution du prix Goncourt à Texaco (attribution de

14. Cf. Gilles Anquetil, " L'Utopie créole de Patrick Chamoiseau ", Le Nouvel Observateur, 27 aout 1992, pp. 5455. Josyane Savigneau, "Chamoiseau, le 'marqueur de paroles' ", Le Monde des livres, 4 sept. 1992. Leila Sebbar, "Un Roman-opéra créole ", Le Magazine littéraire, sept. 1992. Raphaël Sorin, "Saga criolla ", L'Express, sept. 1992. "Les Antilles en quête de racines ", Le Figaro, 5 fév. 1993. Jean-Louis Joubert, "Texaco: Une Polyphonie martiniquaise ", Diagonales, 25, janvier 1993, pp. 18-19. 
divers prix à Chamoiseau et à Confiant, publication de leurs romans par de prestigieux éditeurs parisiens, enthousiasme de la presse parisienne...) ainsi que les conférences « rencontres avec le public » organisées en Martinique manifestent la volonté d'atteindre le grand public martiniquais. En effet, les lecteurs antillais ont toujours attendu que leurs écrivains obtiennent la consécration métropolitaine avant de reconnaître leur importance. Or, et c'est là que le bât blesse, ce public peut-il vraiment " apprécier » Texaco ? Autrement dit, le lecteur revendiqué par Chamoiseau, en compagnie de Barnabé et Confiant dans Éloge de la créolité, est-il celui « programmé » par le texte ? Selon Umberto Eco (1989, pp. 61-83), tout texte littéraire contient en creux l'image de son destinataire, tout auteur " construit " dans son texte, son " lecteur-modèle". Or, le destinataire de Texaco (tel qu'il ressort de l'étude de Delphine Perret, par exemple) n'est pas celui qui peut se déduire de la lecture d'Éloge de la créolité, c'est-à-dire le Martiniquais moyen éduqué. En effet, si nous poussons jusqu'au bout le raisonnement de ce pamphlet, la pratique d'écriture de Chamoiseau et de Confiant devrait viser à se constituer un lectorat martiniquais d'abord puis francophone ensuite. Or, par une ironie de l'histoire, c'est le lectorat métropolitain qui est le plus séduit et enthousiaste. Pour Josyane Savigneau ${ }^{15}$, "loin d'être un renouvellement du roman français par la périphérie, [...] la littérature de Chamoiseau est une affirmation d'appartenance à la culture française dans sa diversité " (p. 5).

Le lectorat antillais est plus partagé. Certains s'enthousiasment comme Serge Médeuf, Georges Desporte, Lisa David et Maryse Romanos qui se fait, elle, carrément lyrique :

Il [Chamoiseau] fomente l'histoire des mots comme l'histoire des hommes sous la pression esthétique de l'écrivain fouettant

15. Cf. "Chamoiseau, le 'marqueur de paroles' ", le Monde des Livres, 4 sept. 1992, repris dans la Sélection hebdomadaire du Monde, semaine du 3 au 6 septembre 1992. 
et fustigeant les canons d'un écrit classique qui se meut dans les hivers-décombres de la folie impérialiste ${ }^{\mathrm{j}}$.

D'autres, comme Xavier Orville et René Ménil, sont plus réticents dans la mesure où ils voient dans Texaco un " doudouisme", nouvelle manière. Ainsi, Ménil interrogé par Le Figaro ironise légèrement, selon le journaliste qui l'a interrogé :

Texaco est un roman qui a de la densité. Mais si Chamoiseau s'était exprimé en français, simplement, cela n'aurait pas nui au livre. Je ne vois pas l'intérêt esthétique ni l'intérêt de la vérité qu'on veut faire passer en ajoutant des mots créoles. On peut se demander si ce n'est pas un peu d'exotisme à usage extérieur".

Par ailleurs, le modèle générativiste à l'œuvre dans le roman postule un lecteur doté d'une culture "encyclopédique " supérieure (maîtrise du créole et du français; connaissance de la culture antillaise, grande culture générale). Comme fait remarquer Eco dans Lector in Fabula,

[...] l'auteur du texte le plus ouvert [...] construit son propre lecteur à travers une stratégie textuelle. Référé à des lecteurs que le texte ne postule pas et qu'il ne contribue pas à produire, le texte devient illisible (plus qu'il ne l'est) ou alors cela devient un autre livre. (pp. 72-73)

Paradoxalement, alors même que Chamoiseau tente de " retrouver » la parole martiniquaise, il crée une langue différente, ni créole, ni française, mais personnelle et placée sous le signe du baroque. Certes, le projet de ce roman (" littérature au lieu vivant est un à-prendre vivant $"$, p. 19) vise ce que Barthes identifie dans

16. « La Créolité de l'En-ville avec Patrick Chamoiseau ", Antilla, 511 (13 nov. 1992), pp. 7-10.

17. "Les Antilles en quête de racines », Le Figaro, 5 fév. 1993, cité dans Antilla, 526 (5 mars 1993), pp. 32-33. 
le Degré zéro de l'écriture comme « la plongée de l'écrivain dans l'opacité poisseuse qu'il décrit " (p. 60). Cependant, il faut signaler que le travail du style, si apparent dans Texaco $^{18}$, contribue à confirmer des automatismes instaurés dans les textes précédents (Chronique, Solibo) et porte en germe le risque d'aboutir à la constitution d'une écriture " consacrée " par les cercles littéraires et les médias parisiens. Dès lors, Chamoiseau court le danger d'aller à l'encontre de son projet (cf. Éloge) en raison même du succès médiatique de Texaco et de se retrouver « prisonnier [...] de mythes formels " (Barthes, 1953, p. 57) qu'il aura lui-même créés, aussi lui reste-t-il à relever le défi de cette relation au langage et à « remonter le courant $"$ des mots ${ }^{19}$. Cependant, malgré cette menace, Texaco illustre la réussite de l'écriture baroque. Celle-ci représente, selon Glissant (1990), la " parole privilégiée » des

cultures composites [...] bâtie[s] dans les marges avec toutes sortes de matériaux qui par nature échappèrent à la patience de la règle [...]. (p. 105)

Le succès de cette écriture, aussi bien dans la littérature antillaise francophone qu'hispano-américaine, est devenu le symbole du métissage culturel et/ou du processus de créolisation qui caractérisent les littératures et les sociétés de notre région. À une époque où, en raison des facilités de communication, les cultures du monde sont

18. Le roman est tout imprégné d'une conscience de l'acte d'écrire et des textes littéraires français qui se manifestent à travers les commentaires des narrateurs et de l'auteur/auditeur/ transcripteur. Cette distanciation vis-à-vis de l'écriture fait de Texaco un roman postmoderne.

19. Cf. Julien Green. Journal, Geuvres complètes (T. 4, 7 février 1931). Paris, Gallimard, Bibliothèque de la Pléiade: « Les mots forment une sorte de courant qu'il faut sans cesse remonter; qui cède à leur entraînement va droit à l'échec, car il devient impossible, après avoir longtemps abusé des mots, de leur faire dire la vérité. » (p. 92) 
mises en contact souvent sans préparation et sans possibilité de distanciation, l'expérience culturelle et littéraire de la Caraíbe contient la promesse ( $\left(\right.$ Babel heureuse $\left.\mathrm{e}^{20} »\right)$ d'un nouveau rapportau-monde basé sur le plurilinguisme.

\section{Références}

BAKHTINE, Mikhaill. (1975). Esthétique et théorie du roman. Paris, Bibliothèque des Idées, NRF/Gallimard, 1978. Traduction française par Daria Olivier.

BARTHES, Roland (1953). Le Degré zéro de l'écriture. Paris, Seuil, Points, 1972.

BERNABÉ, Jean (1978). "Contribution à l'étude de la diglossie littéraire ", ( ( ${ }^{\mathrm{e} r e}$ partie). T.E.D. : Textes, études et documents. Paris, Éditions Caribéennes/G.E.R.E.C.(Groupe d'Études et de Recherches en Espace Créolophone), n 1, pp. 1-16.

(1979). " Contribution à l'étude de la diglossie littéraire " (II' partie). T.E.D. : Textes, études et documents. Paris, Éditions Caribéennes/G.E.R.E.C.(Groupe d'Études et de Recherches en Espace Créolophone), $n^{\circ}$ 2, pp. 103-130.

,Patrick CHAMOISEAU et RaphaëlCONFIANT (1989).

Éloge de la créolité. Paris, Gallimard, édition bilingue, 1993.

CASIMIR, Jean (1991). La Caraibe une et divisible. Port-au-Prince, ONU/CEPALC, Henri Deschamps.

CHAMOISEAU, Patrick, Jean BERNABÉ et Raphaël CONFIANT (1989). Cf. Jean Bernabé, Patrick Chamoiseau et Raphaël Confiant (1989).

20. R. Barthes, le Plaisir du texte (Paris, Seuil, 1973), cité par D. Perret (1995). 
(1994). "Que faire de la parole ? Dans Ia tracée mystérieuse de l'oral à l'écrit ", in Ralph Ludwig (1994), pp. 151158.

CHAUDENSON, Robert (1989). Créole et enseignement du français. Paris, Espaces Francophones/Université de la Réunion/L'Harmattan.

CONDÉ, Maryse, et Madeleine COTTENET-HAGE, dir. (1995). Penser la créolité. Paris, Karthala.

CONFIANT, Raphaël, Patrick CHAMOISEAU et Jean BERNABÉ (1989). Cf. Jean Bernabé, Patrick Chamoiseau et Raphaël Confiant (1989).

Ralph Ludwig (1994), pp. 171-180.

(1994). "Questions pratiques d'écriture créole 》, in

DESOUZA, Pascale (1995). « Inscription du créole dans les textes francophones : De la citation à la créolisation ", in Maryse Condé et Madeleine Cottenet-Hage, dir. (1995), chap. 10, pp. 173-190.

ECO, Umberto (1979). Lector in Fabula : Le rôle du lecteur. (Trad. française, 1985) Paris, Le Livre de Poche, Biblio-Essais, 1989.

FUENTES, Carlos (1990). Valiente mundo nuevo : Epica, utopia y mito en la novela hispano americana. Mexico, Tierta Firme, Fundo de Cultura económica. (Traduction française: Le Sourire d'Érasme)

GLISSANT, Édouard (1990). Poétique de la relation. Paris, Gallimard.

HAGÈGE, Claude (1985). L'Homme de paroles. Chap. II. " Le Laboratoire créole ». Paris, Fayard, Le Temps des sciences, pp. 2941.

KLOSSOWSKI, Pierre (1969). Nietzsche et le Cercle vicieux. Paris, Mercure de France, 1978. 
KUNDERA, Milan (1991a). «The Umbrella, the Night World, and the lonely Moon», The New York Review, 19 déc., pp. 46-50.

multiple ", L'Infini, 34, été.

(1991b) «Beau comme une rencontre

LAROCHE, Maximilien (1991). La Double scène de la représentation: Oraliture et littérature dans la Caraïbe. Sainte-Foy, Québec, GRELCA/Université Laval, Essais 8.

LUDWIG, Ralph, dir. (1994). Écrire la " parole de nuit ": La nouvelle littérature antillaise. Paris, Gallimard, Folio-Essais 239.

PERRET, Delphine (1995). " Lire Chamoiseau », in Maryse Condé et Madeleine Cottenet-Hage, dir. (1995), chap. 9, pp. 153-172.

PRUDENT, Félix Lambert (1981). " Diglossie et interlecte », Langages, 61. Paris, Didier, pp. 13-38.

et Georges Jacques MÉRIDA (1984). « An Langaj kréyòl dimi-panaché... : Interlecte et dynamique conversationnelle », Langage, 74, Paris, Didier, pp. 31-45.

RÉSUMÉ : Littérature et Diglossie : créer une langue métisse ou la "chamoisification" du français dans Texaco de Patrick Chamoiseau - Le succès de Texaco et les commentaires élogieux concernant l'usage du français et du créole par l'auteur invitent à examiner de plus près le jeu linguistique à l'œuvre dans le roman en prenant en compte les positions théoriques avancées par Bernabé, Chamoiseau et Confiant dans Éloge de la créolité (1989). Bien que le roman s'inscrive dans une tradition de l'utilisation littéraire du créole fort ancienne, il est possible d'identifier la spécificité de l'écriture de Chamoiseau. Pratiquant une poétique de « l'inquiétante étrangeté ", il crée un " effet-de-créole 》 grâce auquel il déroute et séduit ses lecteurs hexagonaux aussi bien qu'antillais. Placée sous le signe du Baroque, cette écriture est devenue le symbole du 
métissage culturel et/ou du processus de créolisation, caractéristique des cultures de la région des Caraíbes et riche de la promesse d'un nouveau rapport-au-monde basé sur le plurilinguisme.

\section{ABSTRACT : Literature and Diglossia : The Poetics of French and Creole 'Interlect' in Patrick Chamoiseau's Texaco -} Texaco's success and unanimous praise concerning the mixed use of French and Creole call for a closer examination of the interplay of both languages in the novel. In the light of the theoretical statements made by Bernabé, Chamoiseau et Confiant in Éloge de la créolité / In Praise of Creoleness (1989), one is invited to question the poetics of code-switching ('Interlecte') at work in Texaco. Though the novel belongs to a quite ancient tradition of literary use of Creole, it is possible however to identify specific features in Chamoiseau's writing. As he practices a poetic strategy of 'disquieting strangeness', Chamoiseau creates a 'Creole-like effect' which puzzles and aiso seduces both his Metropolitan French and French Caribbean readers. An expression of the Baroque, this type of writing has become symbolic of cultural miscegenation and/or the Creolization process at work in the Region. This evolution bears in it the promises of a new relation to the world based on multilinguism.

Marie-José N'Zengou-Tayo : Département de français, Université des Indes Occidentales, Campus de Mona, Kingston 7, Jamaïque. 